\title{
Patrones de expansión urbana de las megaurbes latinoamericanas en el nuevo milenio
}

Fermín Cruz-Muñoz. Instituto Politécnico Nacional, Ciudad de México, México.

RESUMEN | Las políticas neoliberales trastocan la urbanización, ahora ceñida a una lógica de mercado donde el sector inmobiliario adquiere un papel cada vez más importante. En este estudio se analiza la evolución de la densidad de población y la morfología del área urbana de las cuatro megaciudades latinoamericanas: Buenos Aires, Ciudad de México, São Paulo y Río de Janeiro. Se encuentra que la expansión urbana es cada vez más dispersa y segmentada espacialmente. Se vincula este patrón de urbanización a las constructoras privadas que localizan conjuntos habitacionales en la periferia metropolitana, siguiendo una lógica de emplazamiento mercantil que fomenta la fragmentación espacial. Se muestra la imperante necesidad de una planificación territorial efectiva para limitar el uso del territorio no urbanizado y asegurar el desarrollo humano en las nuevas periferias urbanas.

PALABRAS CLAVE | expansión urbana, áreas metropolitanas, fragmentación urbana.

ABSTRACT | Neoliberal policies modify urbanization, now adjusted by market laws in which the real estate sector acquires an increasingly important role. In this article, we analyze the evolution of the population density and the morphology of the four megacities of Latin America: Buenos Aires, Mexico City, São Paulo and Rio de Janeiro. The results show that urban sprawl is increasingly dispersed and spatially segmented. This pattern of urbanization is linked with the real estate sector, which built dwellings in the metropolitan periphery from a market location logic that fosters spatial fragmentation. This study expresses the imperative need of effective territorial planning in order to limit the use of the non-urbanized territory and to ensure human development in the new urban peripheries.

KEYWORDS | urban sprawl, metropolitan areas, urban fragmentation.

Recibido el 23 de abril de 2019, aprobado el 3 de septiembre de 2019.

E-mail: facruzm@ipn.mx 


\section{Introducción}

Según estimaciones del Banco Mundial, cerca de mil millones de personas a nivel mundial habitan en espacios con calidad deficiente o sin infraestructura básica (Robinson, Scott \& Taylor, 2016, p. 40), y ello contrariamente al principio según el cual el entorno debe satisfacer las necesidades objetivas y subjetivas de una sociedad y de sus individuos. Es en este marco que Zulaica y Celemín (2008, p. 130) vinculan la habitabilidad con el concepto de calidad de vida. De hecho, como señala Martínez (2014), los patrones de urbanización contribuyen a definir un modo de vida, comportamientos y prácticas sociales (p. 9). Y en palabras de Heidegger (1994), la esencia del hábitat es dejar habitar (p. 140).

Después de la Segunda Guerra Mundial, se identificó un nuevo proceso de dispersión urbana denominado sprawl (Nechyba \& Walsh, 2004, p. 177), en el cual se reconoce la forma en que opera la lógica del libre mercado en el sector inmobiliario. Al respecto, Harvey y Clark (1965, p. 2) aseguran que las decisiones independientes de los grandes monopolios orientadas a ofrecer vivienda generan discontinuidad urbana, proceso que, a su vez, se encuentra estrechamente vinculado a un cambio de visión gubernamental respecto a la planeación. Peiser (2001, p. 276), por su parte, identifica un abandono de las políticas de ordenamiento territorial en favor de la meta de vincular las zonas habitacionales del empleo y las del comercio. Aunado a ello, la política pública favorece la vivienda unifamiliar en las áreas suburbanas, lo que se refleja en un decremento en la densidad de población.

Se ha identificado una serie de efectos que se relacionan con la fragmentación funcional de la estructura urbana (Peiser, 2001, p. 279) y la deficiente accesibilidad entre sus distintos segmentos (Ewing, 2008, p. 521). Este último problema se radicaliza en el caso del desajuste espacial entre los residentes de la zona periférica y el empleo, situación que tiende a generar una saturación de las vías de comunicación, altos niveles de contaminación por el uso del vehículo automotor, pérdida de equipamiento al aire libre y dotación desigual de servicios. Todo ello, sumado, agudiza la segregación residencial y la pobreza (Nechyba \& Walsh, 2004, p. 178).

Mucha de la literatura en estas materias se ha construido en torno a los procesos de urbanización anglosajona. Si bien se han realizado aproximaciones en ciudades latinoamericanas (Herzog, 2015; Inostroza, Baur \& Csaplovics, 2013; Pradilla, 2017), poco se ha hecho para cuantificar este nuevo patrón de expansión disperso y sus efectos en las urbes de mayor magnitud en Latinoamérica, en el contexto del impulso inmobiliario. Por ello, este estudio es un esfuerzo por conjuntar estudios locales y determinar condiciones convergentes, e identificar diferencias, entre las megaurbes latinoamericanas.

El objetivo de este trabajo es dar cuenta del proceso social de construcción del hábitat en la periferia metropolitana ante un contexto de oferta inmobiliaria de masivos conjuntos habitacionales. Con tal fin se realiza un análisis comparativo del proceso de expansión urbana de las cuatro megaurbes latinoamericanas: Ciudad de México, São Paulo, Río de Janeiro y Buenos Aires. Este estudio morfológico y de ocupación del suelo permite elaborar proyecciones de densidad poblacional y demanda habitacional, avanzar en la comprensión de los procesos de producción 
mercantil del espacio, y esbozar los retos que enfrentan los habitantes para lograr habitabilidad mediante la apropiación del espacio.

El punto de partida es, entonces, que la construcción de hábitat representa un problema social. Como señala Zamanillo (2014):

Cuando la falta de alojamiento de las clases trabajadoras es generalizada, la habitación se transforma en "problema social". Dado que la habitación urbana depende de medios de consumo o soportes materiales que solo existen bajo la forma colectiva (el saneamiento, agua y electricidad, tipo de construcción y su localización, etc.), o sea bienes y servicios indivisibles, puede decirse que el problema habitacional se torna doblemente social. (p.140)

\section{Metodología: los patrones de urbanización y sus implicaciones en el hábitat}

La morfología de las ciudades es determinada por la distribución de las actividades urbanas. Tradicionalmente se reconocen tres tipos básicos de expansión dispersa de las zonas urbanas. El primero se describe como un crecimiento con muy baja densidad, donde la demanda de suelo es abrumadora en función de la poca población que lo habita (Harvey \& Clark, 1965, p. 2). El segundo se describe como franjas urbanas creadas a lo largo de las vías de comunicación importantes, lo que deja grandes intersticios sin urbanizar. El tercero es el "salto de rana", donde se crean asentamientos discontinuos.

Actualmente las grandes metrópolis se caracterizan por un creciente proceso de especialización del espacio urbano, donde se incrementa la fragmentación en las dimensiones espacial, social y política (Prévôt, 2001, p. 34). Esta dispersión de las actividades urbanas -entre ellas, las económicas- ha generado un nuevo patrón, que Lang (2003) denominó edgeless cities, donde no es posible identificar aglomeraciones importantes o la generación de subcentros en las metrópolis.

Con el objetivo de determinar los patrones de urbanización de las megaurbes, se estudiará la tendencia metropolitana de expansión urbana a partir de una revisión de la evolución de la densidad de población y del grado de compacidad geométrica. Al respecto, el índice de compacidad se define a través de la morfología de los polígonos urbanos y su grado de regularidad geométrica, entendiendo que la figura más compacta es el círculo, dada su máxima área posible en función de su perímetro. También se considera la cantidad de polígonos que forman el área metropolitana, por lo que, a mayor cantidad de polígonos, disminuye el índice de compacidad. Los valores posibles oscilan entre uno y cero, donde 1 representaría una circunferencia y, por ende, la máxima compacidad posible; y 0 , la completa fragmentación e irregularidad geométrica de los polígonos. La fórmula para la estimación del índice se expresa de la siguiente forma:

$$
I C=\frac{\sum_{i} 2 \pi^{\sqrt{s_{i} \div \pi}} / p_{i}}{N^{2}}
$$

donde $I C$ es el índice de compacidad, $p_{i}$ es el perímetro del polígono $i, s_{i}$ es el área del polígono $i$ y $N$ es el total de polígonos (Jingnan, Lu \& Sellers, 2007). 
El periodo de análisis que se abarca en este estudio es esencialmente de 2000 a 2010, aunque se realizan proyecciones para 2020. Se determinará la presencia de la periurbanización o de la suburbanización, entendiendo que la primera describe una discontinuidad en el crecimiento urbano, mientras la segunda implica un fenómeno de propagación de una ciudad hacia la tierra rural en la periferia. La periurbanización también es conocida como exourbanización (Guillain, Le Gallo \& Boiteux-Orain, 2006, p. 2076).

Para realizar el análisis se consideraron fuentes estadísticas y geográficas. Las fuentes estadísticas utilizadas son los Censos Demográficos de 2000 y 2010 del Instituto Brasileiro de Geografía e Estadística (IBGE) para el caso de Río de Janeiro y São Paulo; los Censos Nacionales de Población, Hogares y Vivienda de 2001 y 2010 del Instituto Nacional de Estadística y Censos (INDEC) para el caso de Buenos Aires; y los Censos de Población y Vivienda 2000 y 2010 del Instituto Nacional de Geografía y Estadística (INEgi) para la Ciudad de México.

Las variables que se utilizaron para analizar el proceso de expansión metropolitana fueron la población de cada zona metropolitana y la dotación de servicios básicos a las viviendas localizadas en la periferia urbana. Se consideró como periferia los municipios conurbados de Río de Janeiro (17 municipios), São Paulo (38 municipios) y de la Ciudad de México (59 municipios), así como los 24 partidos del Gran Buenos Aires. Respecto a la información cartográfica, se utilizaron los marcos geoestadísticos de la IBGE, INDEC e INEGI. Esta información se complementó a partir de fotointerpretación derivada de fotografías aéreas.

A partir de estudios específicos de cada megaurbe, se analizan los detalles de las nuevas dinámicas de expansión urbana dictadas por la política mercantil encabezada por el sector inmobiliario. Ello para identificar las similitudes y diferencias en la construcción del hábitat, que derivan en nuevos desafíos para la población que, en muchas ocasiones, no logran superar, lo que genera un deterioro en el hábitat periférico. Este estudio se centrará esencialmente en esta etapa de cambio de siglo una vez iniciada la segunda década del Xxi, donde ya es posible palpar los efectos en el territorio.

\section{Creciente dispersión urbana como reto en la construcción del hábitat}

Actualmente, las grandes ciudades en Latinoamérica constituyen un importante nodo de aglomeración de población, pues $14 \%$ de la población urbana se localiza en ciudades de más de 10 millones de habitantes, en comparación con el $9 \%$ mundial (onU-Hábitat, 2012, p. 25). Estas megaurbes se caracterizan por su gran primacía en los sistemas urbanos de sus naciones, y alcanzan extensiones territoriales de hasta miles de kilómetros cuadrados (Picorelli, Barros, Tomas, \& Molle, 2009) (tabla 1). 


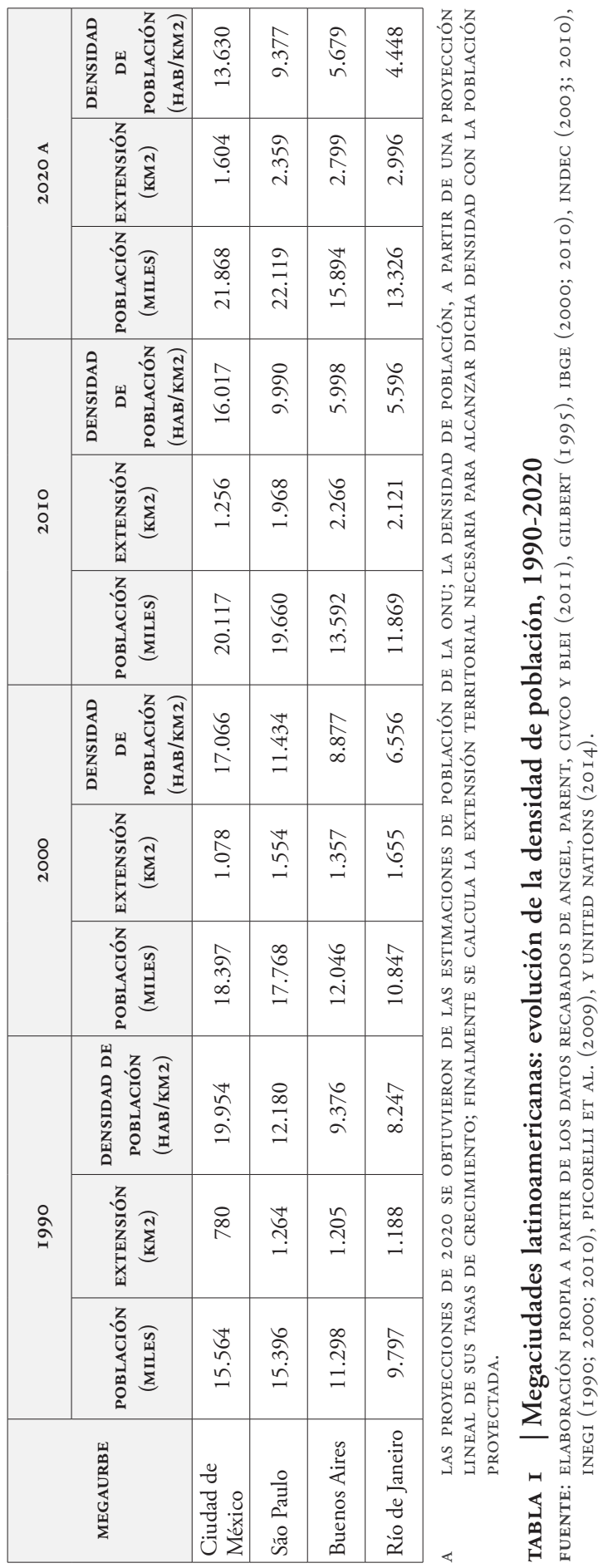


Es claro que la tasa de crecimiento media anual (TCMA) de la población urbana ha disminuido en términos porcentuales como consecuencia del decremento de los procesos migratorios de la población rural y de la reducción de la tasa de fecundidad. En contrapartida, la expansión de las ciudades es creciente. En el tabla 1 se puede observar el incremento de la extensión territorial de las megaurbes latinoamericanas, a tal grado que las densidades de población están en claro declive. Por un lado se tiene la Ciudad de México, que muestra durante el periodo de 1990 a 2010 la mayor densidad de población, en contraste de Río de Janeiro, la megaurbe menos poblada pero también la más dispersa. Ello evidencia que la magnitud de la urbe no deriva en una mayor dispersión, siendo otros factores, que se revisarán en la siguiente sección, los motivadores de dicho fenómeno.

No obstante, es claro que en las cuatro megaciudades se presenta un proceso de uso extensivo del suelo con una reducción sostenida de la densidad de población. A partir de esta tendencia es que se prevé que, para 2020, la densidad de las cuatro áreas metropolitana será prácticamente la mitad de la registrada en 1990. Ello significa que la velocidad de expansión urbana es claramente mayor que el incremento poblacional, tal y como se puede ver en el tabla 2.

\begin{tabular}{|c|c|c|c|c|c|c|c|}
\hline \multirow{2}{*}{ MEGAURBE } & \multicolumn{2}{|c|}{$\begin{array}{l}\text { CRECIMIENTO } \\
\text { POBLACIONAL } \\
\text { (PORCENTAJES) }\end{array}$} & \multicolumn{2}{|c|}{$\begin{array}{c}\text { TASA DE EXPANSIÓN } \\
\text { URBANA } \\
\text { (PORCENTAJES) }\end{array}$} & \multicolumn{3}{|c|}{$\begin{array}{c}\text { DEMANDA DE SUELO POR } \\
\text { HABITANTE } \\
\left(\mathrm{HAB} / \mathrm{M}^{2}\right)\end{array}$} \\
\hline & $\begin{array}{l}1990- \\
2000\end{array}$ & $\begin{array}{l}2000- \\
2010\end{array}$ & $\begin{array}{l}1990- \\
2000\end{array}$ & $\begin{array}{l}2000- \\
2010\end{array}$ & I990 & 2000 & 2010 \\
\hline $\begin{array}{l}\text { Ciudad de } \\
\text { México }\end{array}$ & 1,69 & 0,90 & 3,29 & 1,54 & 50,1 & 58,6 & 62,4 \\
\hline São Paulo & 1,44 & 1,02 & 2,09 & 2,39 & 82,1 & 87,5 & 100,1 \\
\hline Buenos Aires & 0,64 & 1,21 & 1,20 & 5,26 & 106,7 & 112,7 & 166,7 \\
\hline $\begin{array}{l}\text { Río de } \\
\text { Janeiro }\end{array}$ & 1,02 & 0,90 & 3,37 & 2,52 & 121,3 & 152,5 & 178,7 \\
\hline
\end{tabular}

TABLA 2 | Megaciudades latinoamericanas: tasas de crecimiento anual de la población y del área urbana, 1990-2010

FUENTE: ELABORACIÓN PROPIA A PARTIR DE LOS DATOS DE LA TABLA I

Si bien en las cuatro megaurbes se tienen tasas similares de crecimiento poblacional, que oscilan entre 0,64 y 1,69\%, la diferencia y ritmo de expansión de la superficie urbana en todas ellas son altos. Por un lado, se tiene ciudades cuya velocidad de expansión va en clara disminución, como es el caso de la Ciudad de México, pues ha pasado de 3,29 a 1,54\%, mientras que Buenos Aires se encuentra en un proceso inverso, con la mayor tasa de todas, 5,26\%. Asimismo, las ciudades brasileńas mantienen un comportamiento más estable. No cabe duda de que, en todos los casos, la construcción de nuevos complejos residenciales, áreas industriales o nuevos asentamientos irregulares, llega a ser dos o tres veces superior al incremento poblacional, lo que se refleja en la disminución de la densidad urbana. 
Dado el patrón señalado, y a pesar de un proceso de estabilización del crecimiento de las grandes urbes, se da en ellas un creciente consumo de suelo rural. En promedio, las megaciudades latinoamericanas pasaron de una densidad urbana de 117 hab/ha en 1990 a 85,7 hab/ha en 2010, o -lo que es lo mismo-, de una demanda de suelo de $85,2 \mathrm{~m}^{2}$ por habitante a $116,7 \mathrm{~m}^{2}$ por persona en veinte años. Esto se traduce, en términos reales, en un incremento de cientos de kilómetros cuadrados de suelo natural a urbano, lo que se expresa en una dispersión intensiva de las urbes y una depredación del medio natural circundante. Destacan los casos de Buenos Aires y Río de Janeiro, las ciudades en que más suelo se demanda por habitante y que tuvieron un incremento de 106,7 y $121,3 \mathrm{~m}^{2}$ en 1990 , a 166,7 y $178,7 \mathrm{~m}^{2}$ en 2010, respectivamente; más del doble que la Ciudad de México y muy por encima de São Paulo.

La situación descrita significa un reto para el proceso de planeación y construcción del hábitat de la nueva población urbana, pues no necesariamente se expresa en un incremento de espacio urbano que favorecería la habitabilidad. Más bien, la alta dispersión al interior de las ciudades promueve la fragmentación perceptual y dificulta la apropiación de un espacio demasiado extenso que -como se verá más adelante- no presenta las condiciones adecuadas para el desarrollo de las actividades de la población. La dispersión urbana representa un incremento de costos de introducción de infraestructura, así como de distancias, tiempo y costos de traslados desde los centros habitacionales a los centros de trabajo, estudio y servicios de equipamiento. Como consecuencia, los costos de urbanización y dotación de los componentes básicos para la construcción del hábitat aumentan y los recursos de los gobiernos locales se hacen insuficientes, dado el ritmo de expansión desperdigada de asentamientos.

No obstante lo anterior, las ciudades no solamente muestran una reducción de su densidad de población, sino que también presentan un nuevo patrón morfológico de expansión que radicaliza aún más la construcción del hábitat metropolitano. En la siguiente sección se abordará, desde una visión metropolitana, las condiciones de urbanización de las nuevas periferias de las megaciudades latinoamericanas y cómo se construyen los nuevos hábitats a partir de la lógica mercantil.

\section{Discontinuidad espacial ante la construcción mercantil de las nuevas periferias urbanas}

Con la implementación de las políticas neoliberales, la mayoría de las naciones latinoamericanas modificó sus políticas de desarrollo urbano para satisfacer la creciente necesidad de vivienda. En ese sentido, la visión del Estado en cuanto a la gestión urbana se fue transformando, para pasar de un enfoque racionalista (Nivón, 2016, p. 10) a uno empresarial. Ello implica que ahora el "libre mercado" define las reglas de ordenamiento y expansión de las metrópolis, donde la maximización de utilidades les otorga a las empresas inmobiliarias un rol central en los procesos de urbanización, en detrimento del papel del sector público como gestor y regulador (Perahia, 2009). 

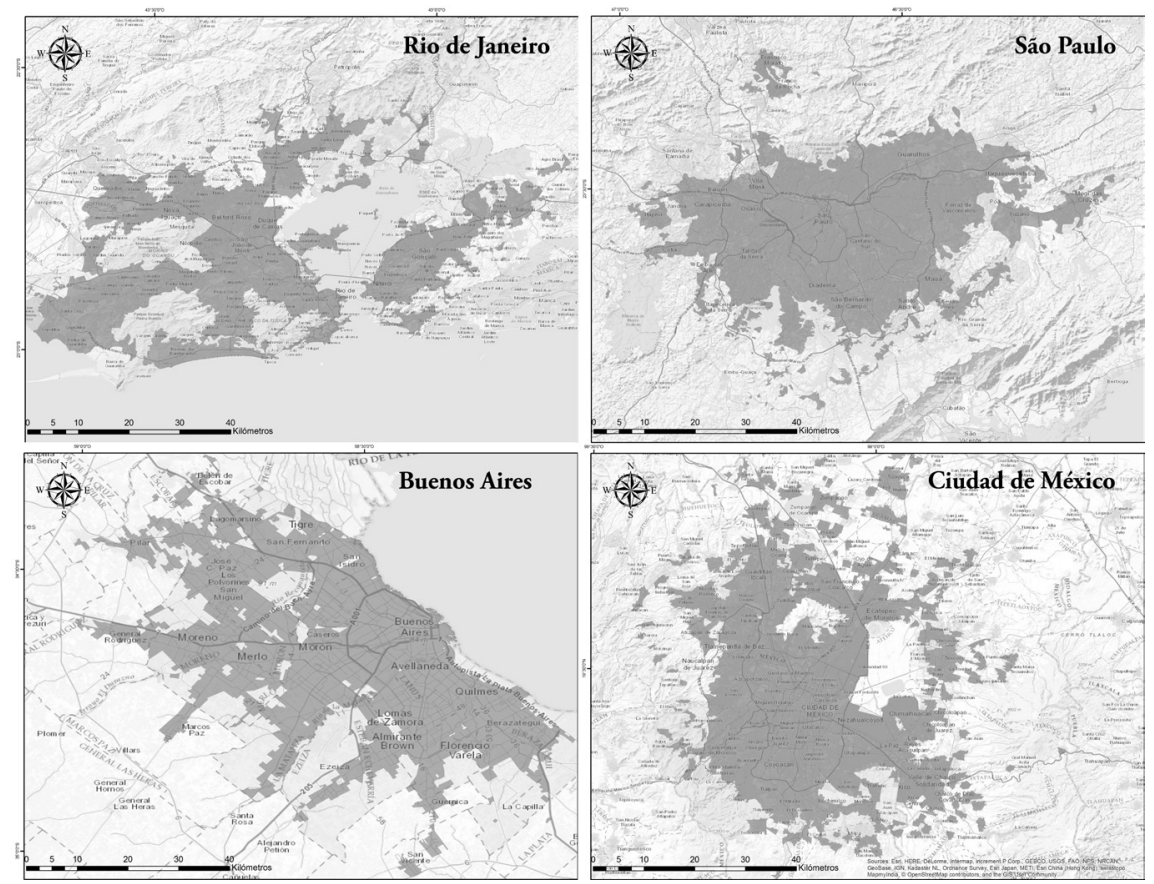

FIGURA I | Megaciudades latinoamericanas: morfología de las áreas urbanas, 2010 FUENTE: ELABORACIÓN PROPIA A PARTIR DE LOS MARCOS GEOGRÁFICOS DE IBGE (2OIO), INDEC (2OIO) E INEGI (2OIO)

Desde esta nueva lógica de urbanización, se ha modificado el patrón morfológico dominante de las megaciudades latinoamericanas, que han pasado de una urbanización con límites relativamente identificables -herencia de lo que De Mattos (1998, p. 746) denomina el periodo desarrollista-, a una región urbana caracterizada por una metropolización expandida, que ha dado la pauta para la generación de conceptos como "exopolis" (Soja, 1992) o "postborder city" (Dear \& Leclerc, 2013). Con la transición hacia nuevo milenio, las megaciudades latinoamericanas, al igual que otras megaurbes del mundo, se caracterizan por una expansión difusa cuyo resultado puede describirse como un archipiélago urbano (De Mattos, 1998, p. 746).

Con el inicio de la segunda década de este milenio, la reterritorialización que implican los procesos señalados se manifiesta ya claramente en la morfología de las áreas metropolitanas de las cuatro megaciudades (figura 1). En los cuatro casos se puede identificar un polígono principal de mayor magnitud y población, circundado por una serie de micropolígonos que representan las áreas urbanizadas y próximas a conurbarse, sin una continuidad urbana con el polígono principal. Esta morfología se expresa en los índices de compacidad, que ayudan a cuantificar el grado de fragmentación geométrica de las áreas metropolitanas (tabla 3). 


\begin{tabular}{|l|c|c|c|}
\hline \multicolumn{1}{|c|}{ MEGAURBE } & $\begin{array}{c}\text { ÍNDICE DE } \\
\text { COMPACIDAD }\end{array}$ & $\begin{array}{c}\text { NÚMERO DE } \\
\text { POLÍGONOS }\end{array}$ & $\begin{array}{c}\text { ÁREA DEL POĹGONO } \\
\text { PRINCIPAL (\%) }\end{array}$ \\
\hline Ciudad de México & 0,0076 & 74 & 80,6 \\
\hline São Paulo & 0,0042 & 156 & 84,8 \\
\hline Buenos Aires & 0,0314 & 22 & 96,1 \\
\hline Río de Janeiro & 0,0069 & 88 & 67,3 \\
\hline
\end{tabular}

TABLA 3 | Megaciudades latinoamericanas: grado de compacidad, 2010

FUENTE: CÁlCULOS PROPIOS A PARTIR DE LA CONSTRUCCIÓN DE UN SISTEMA DE INFORMACIÓN GEOGRÁFICA BASADO EN LA CARTOGRAFÍA DE IBGE (20IO), INDEC (20IO) E INEGI (20IO)

Como se puede apreciar, las cuatro ciudades tienen un índice de compacidad muy bajo, resultado principalmente de la existencia de numerosos polígonos urbanos circundantes al área urbana principal. La megaciudad con menor índice de compacidad es São Paulo, y esto se entiende por la gran cantidad de pequeños asentamientos en torno al polígono central. En segunda instancia se encuentra Río de Janeiro, cuyo polígono principal representa solamente $67,3 \%$ del total del área metropolitana, ello como consecuencia de las condiciones topográficas del territorio de emplazamiento. Aunado a ello, también tiene una gran cantidad de asentamientos de diversos tamaños, que generan un índice de compacidad bastante bajo. En el caso de Buenos Aires se observa una mayor compacidad en relación al resto de las megaurbes. Esto se explica esencialmente por el menor números de asentamientos aislados y la proporción que representa el polígono central respecto de la ciudad, de $96,1 \%$, el más alto de todos. Ello muestra que la morfología de Buenos Aires todavía conserva fuertes reminiscencias del proceso de urbanización y expansión tradicionalmente llamado "mancha de aceite" (Ducci, 1998), pero en función de su ritmo de expansión poco falta para que adquiera un patrón fragmentado similar al de las otras megaciudades.

Esta recomposición territorial de las periferias metropolitanas se vincula directamente con la nueva lógica de urbanización, donde el mercado y la maximización de utilidades dictan el emplazamiento y los procesos de ocupación del suelo. Es decir, la expansión periférica se explica en gran medida por los desarrollos inmobiliarios (Nivón, 2016, p. 10). ${ }^{1}$

En el marco de este proceso, la periferia urbana de las grandes ciudades latinoamericanas se ha convertido en un área de dominante uso habitacional, desde la cual la población tiende a trasladarse a sus centros de trabajo, situación manifiesta de un desajuste espacial vivienda-trabajo (Rodríguez, 2008). Como resultado, se

1 Cabe resaltar que la intensidad de esta tendencia de construcción del hábitat periférico es diferente en cada país. En México, las modificaciones a la política de vivienda se iniciaron en el sexenio de Salinas de Gortari (1989-1994), con la creación del Programa Especial para el Fomento y Desregulación de la Vivienda, que implementaba políticas enfocadas en la promoción de la inversión privada para la construcción de vivienda. con limitada función financiera de los organismos públicos (Maya et al., 2013, p. 43). También en los noventa se adoptó en Argentina la lógica de participación privada en la construcción de vivienda (Prévôt, 2002, p. 32). En Brasil existe una legislación que busca proteger las comunidades pobres para evitar que se vean sometidas a la lógica de las desarrolladoras inmobiliarias (Robinson et al., 2016, p. 50). 
puede hablar de un hábitat fragmentado, donde la vida cotidiana y la apropiación del espacio se dan a un nivel multiescalar y multidimensional. En ese sentido, en la periferia de las metrópolis predomina un hábitat residencial, que constituye su eje fundamental, pues impulsa la transformación de la comunidad (Carnevali \& Trujillo, 2010, p. 26). No obstante, no se debe caer en el error de diversas instituciones públicas en el contexto latinoamericana, que conciben al hábitat solo a través de la casa habitación, sin considerar el contexto urbano. Tal es el origen de una visión viviendista, que ha guiado el diseño de estrategias adaptadas como mecanismo para la obtención de recursos de las empresas inmobiliarias en el marco de las políticas neoliberales. Bajo la lógica de mercado y de maximización de utilidades que este situación representa, y dado que las empresas inmobiliarias no siempre se preocupan por aportar condiciones urbanas en favor de la habitabilidad (Jiménez, 2013, p. 199), la ciudad tiende a crecer hacia áreas definidas por la mayor rentabilidad de los proyectos inmobiliarios. La dinámica de expansión de las megaciudades de América Latina obedece así a un esquema basado en la multiplicación del hábitat a partir de los intereses de las empresas inmobiliarias (De Mattos, 2008; Hidalgo, Borsdorf, Zunino, \& Álvarez, 2008). Las inmobiliarias se encargan también de administrar la infraestructura vial y el "nuevo espacio público", expresado principalmente por los centros comerciales, proceso aunado al incremento de los conjuntos habitacionales privados.

En consonancia con el proceso señalado, los complejos inmobiliarios que se incorporan a la morfología de las periferias metropolitanas se realizan al margen de una perspectiva metropolitana de desarrollo, de forma inconexa y fragmentada (De Mattos, 2006, p. 66). Ese trata de un modelo de desarrollo urbano que responde de manera puntual a las múltiples demandas de los mercados inmobiliarios, sin mayor intervención de las instituciones públicas, y que se orienta a adecuar las estructuras urbanas en función de las nuevas dinámicas sociales, políticas y económicas. A escala de la ciudad, la discontinuidad espacial y fragmentación espacial que genera este modelo se expresan en una fragmentación social llena de vacíos y desarticulaciones territoriales y sociales, que tienden hacia la individualización (Prévôt $\&$ Cattaneo, 2008, p. 86), hacia la falta de aquella cohesión e integración social que pudieran favorecerse en la construcción de hábitats socialmente desarrollados, no mercantilmente construidos y segmentados de la traza urbana.

En la figura 2 se puede observar casos particulares de cada una de las periferias metropolitanas de las cuatro urbes en cuestión. En los cuatro casos se identifican patrones de "saltos de rana" que generan conjuntos habitacionales construidos por el sector inmobiliario. Para el caso de las ciudades brasileñas, Río de Janeiro tiene una expansión urbana que alcanza el municipio de Nova Iguaçu, donde se observan varias unidades habitacionales, como "Residencial Nice", cuyo diseño y traza de calles no se integran a la existente y se localizan en los intersticios de los poblados. Respecto de São Paulo, el conjunto habitacional Valle Verde, localizado en el municipio conurbado de Cotia, se desintegra de la urbanización aledaña mediante una franja de área verde que formalmente no es un espacio público aprovechable por los habitantes del conjunto habitacional ni por los pobladores. Caso particular se identifica en la Ciudad de Buenos Aires, pues en el partido de Presidente Perón 
se localizan diversos conjuntos habitacionales terminados y otros en proceso de construcción, ubicados en un área completamente aislada de la zona urbana. Nuevamente se identifican fraccionamientos cerrados, sin la posibilidad futura de integrarse a un entramado vial, y ubicados en un emplazamiento que motiva la fragmentación espacial. Mismo fenómeno ocurre en el municipio de Huehuetoca, periferia norte de la Ciudad de México, donde masivos conjuntos habitacionales, como Paseos de la Pradera, se encuentran en una zona completamente aislada de la zona urbana. En todos los casos, es clara la ausencia de una conectividad adecuada con el resto de la urbe.

En el marco de este proceso de urbanización fragmentada, las ciudades latinoamericanas se expanden con viviendas que, en el mejor de los casos, están dotadas de infraestructura y servicios, pero sin constituir un verdadero tejido urbano interconectado (onU-Hábitat, 2012, p. 36). Sin una visión integradora, en las nuevas periferias metropolitanas se incrementa la segregación residencial socioespacial (Rodríguez \& Arriagada, 2004), agudizando aún más la inequidad urbana. Con ello se refuerza el proceso según el cual en las periferias urbanas se generan hábitats de pobreza cuyos habitantes, ante la deficiencia de las condiciones materiales de infraestructura y equipamiento, se ven relegados de las ventajas que ofrecen las grandes metrópolis.
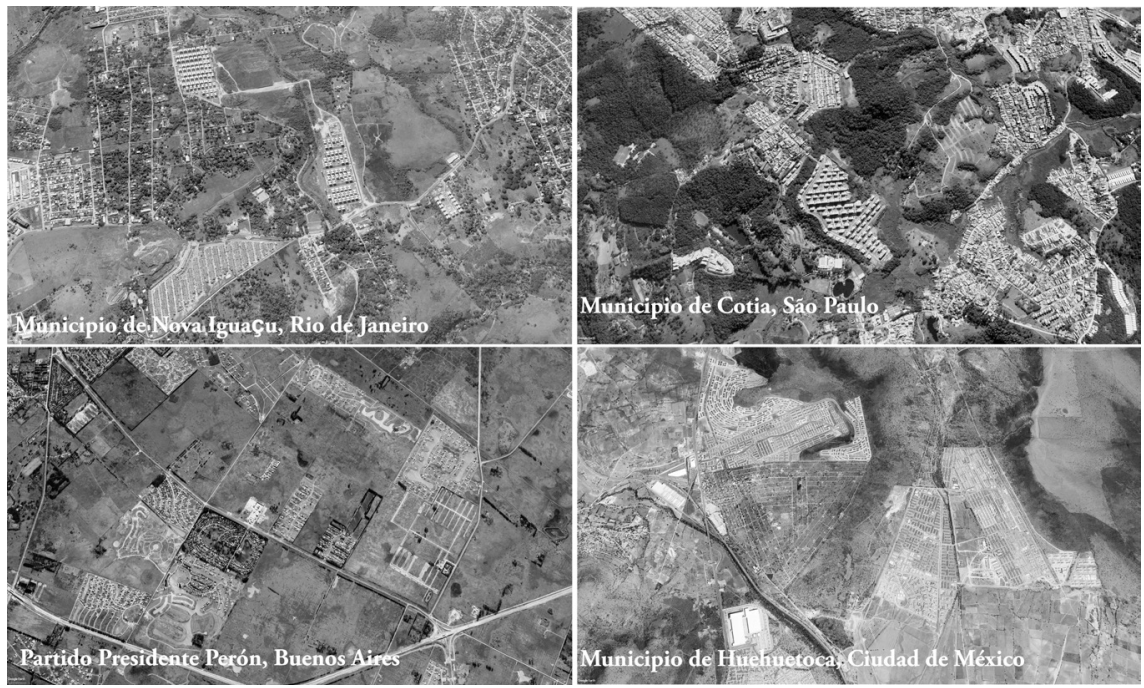

FIGURA 2 | Megaciudades latinoamericanas: patrón fragmentado de expansión urbana, 2018

FUENTE: ELABORACIÓN PROPIA A PARTIR DE FOTOGRAFÍAS DISPONIBLES EN GOOGLE EARTH

Ante la nueva lógica que prioriza las leyes del mercado de suelo como mecanismo que orienta el crecimiento urbano, prolifera la especulación de las empresas inmobiliarias y de los propietarios de las tierras circundantes a los nuevos conjuntos. Estos últimos retiran del mercado sus parcelas, con el afán de incrementar su valor como consecuencia de la introducción de infraestructura en sus alrededores y por 
el incremento de la demanda de suelo en dichas áreas de la ciudad. Al generarse una falsa escasez de suelo y ante las tendencias de expansión urbana, el valor de sus predios se incrementa. La especulación inmobiliaria fomenta que fragmentos de la tierra se mantengan sin construcciones, ocasionando una expansión urbana con grandes áreas libres y bajas densidades de población y de uso de suelo (Titman, 1985, p. 513).

En la perspectiva neoliberal, el hábitat también es reinterpretado, entendiéndoselo como el "subsistema de oferta" de vivienda, recursos y servicios urbanos que, en teoría, están disponibles para la sociedad urbana. Su contraparte es el habitar, que se entiende como el "subsistema de demandas" de vivienda, recursos y servicios urbanos indispensables para el desarrollo de la vida cotidiana de la población (Zulaica \& Celemín, 2008, p. 132). Como consecuencia, otorgar un espacio habitable se interpreta esencialmente como la satisfacción de las demandas sociales de cobijo desde la perspectiva del consumo. De esta forma, se asocia la habitabilidad a la satisfacción de necesidades mediante el consumo (Arcas, Pagès, \& Casals, 2011, p. 84). En esta línea, en los actuales procesos de urbanización y de construcción de los espacio de vida, el hábitat está determinado por la oferta de bienes, servicios y condiciones que pudiera llegar a abastecer el sector privado (Zulaica \& Celemín, 2008, p. 135). En este escenario, se limita el papel de las instituciones gubernamentales en cuanto a la dotación de equipamiento y espacio de convivencia, elementos necesarios para la construcción de un hábitat adecuado a la realización de las prácticas sociales, ahora restringidas a meras prácticas de consumo.

En los procesos de construcción de la ciudad suburbana se ha pasado desde la urbanización informal -con deficiencias en cuanto a la construcción de un hábitat adecuado, por la ausencia de un diseño urbano que contemplara todos los servicios básicos-, a una nueva lógica de urbanización que fomenta la proliferación de espacios formalmente construidos, pero con la misma deficiencia de espacios públicos que dificulta tanto la apropiación del territorio por los residentes, como la construcción de un hábitat propio (Brites, 2012; Esquivel, Maya, \& Cervantes, 2005; Thuillier, 2005). Se mantiene la condición según la cual, al vivir en un barrio o conjunto habitacional precario, se reducen el acceso y las oportunidades de empleo, educación y servicios (Brites, 2012; Cruz \& Isunza, 2017; Maya \& Cervantes, 1999), solo que ahora bajo la dinámica del libre mercado, donde las empresas no consideran a este segmento de la población como mercado potencial para ofrecerles servicios de equipamiento.

\section{Cualidades de habitabilidad en la periferia dispersa}

La dispersión creciente a escala metropolitana tiene sus implicaciones en los espacios de vida de la población. Entre ellas, por ejemplo, la necesidad de realizar traslados mayores para acceder a destinos más desperdigados genera una percepción del espacio indiferente, cuya apropiación se dificulta como consecuencia de concebir el territorio como zona de circulación en lugar de uso.

Los nuevos hogares formados a partir de la población residente y de las familias inmigrantes tienden a establecer su residencia en municipios periféricos a la urbe 
(onU-Hábitat, 2012, p. 25). Esta tendencia ha enfatizado la formación de ciudades satélite y ciudades dormitorio, que representan núcleos urbanos emplazados cerca del polígono principal, estrechamente dependientes de él por empleo y servicios de equipamiento. Es así que muchos de estos nuevos asentamientos periurbanos, aunque pueden tener cierta autonomía funcional por la dotación de servicios de consumo básico como educación, salud y comercio, están estrechamente vinculados a la ciudad principal (onU-Hábitat, 2012, p. 34).

Las periferias de urbes en general presentan una mezcla de asentamientos rurales con los nuevos asentamientos urbanos. Lo que en tiempos pretéritos fueron espacios rururbanos, ahora están sometidos a cambios en su configuración territorial y en el perfil socioeconómico del habitante, alienando la cultura rururbana local hacia una visión más urbana (Hernández, Ocón, \& Guillén, 2009). Este proceso es definido por Augé (1992, p. 44) como la sobremodernidad, que fomenta la construcción de "no lugares", donde se carece de simbolismo y memoria y predomina la individualidad solitaria, manteniendo ausente lo que llamamos sociedad.

Durante la segunda mitad del siglo xx y época de mayor crecimiento urbano en Latinoamérica, para la mayoría de la población los asentamientos informales eran la única forma de obtención de suelo y posterior autoconstrucción de la vivienda. El espacio urbano así construido -considerado "popular"- se ha materializado en el territorio a través de actividades autogestionarias de los residentes, destinadas a mejorar, consolidar e integrar el espacio inmediato mediante el uso de su propio capital humano, económico y social (Hernández, 2008, p. 110). Más allá de sus carencias, este espacio urbano enseña cuán primordial es que un lugar habitable ofrezca las posibilidades de creación y adaptación de los individuos para su apropiación (Delabarre \& Marry, 2012), pues solo ante la intervención activa del habitante urbano en la construcción de su hábitat, se logra la "producción social de hábitat". Se refiere esto a la modalidad integral de acceso al suelo que permite a los individuos y comunidad producir su hábitat de forma que controlen las decisiones fundamentales mediante procesos de gestión efectivos a partir de políticas, estrategias, legislación y asesorías que faciliten ese proceso (Corzo, 2012, p. 147).

En un sentido contrario, la alta dispersión de las nuevas áreas urbanas da como resultado la creación de territorios con equipamientos y servicios insuficientes (Pradilla, 2017, p. 40) y muy bajos niveles de calidad de vida. Además, existe en esta modalidad de construcción urbana una fuerte tendencia hacia la privatización de los espacios públicos. Aunque en los segmentos de la urbe donde residen los estratos socioeconómicos medios y altos este proceso de apropiación del espacio público se desarrolla de manera intensiva a partir de la construcción de centros comerciales y recreativos, es en las colonias, fraccionamientos y barrios de los sectores de menores recursos donde el poco espacio público se encuentra amenazado por diversos agentes que fomentan su privatización a través de la construcción de viviendas y otro tipo de edificios. Por otra parte, aquellos espacios que forman la base territorial para la convivencia comunitaria, los espacios públicos, también están en riesgo de abandono ante la falta de su apropiación por los habitantes. Es fundamental subrayar que el espacio público es tan necesario como el espacio privado para el desarrollo de las sociedades urbanas (Hernández, 2008, p. 111). Y ello, por supuesto, sin olvidar 
la provisión de servicios. Porque aunque las zonas urbanas de América Latina y el Caribe ya tienen cobertura suficiente -por ejemplo- en materia de abastecimiento de agua, que alcanza al 97,5\% de los habitantes, cerca de $25 \%$ la obtiene por medios precarios y, en ocasiones, informales o clandestinos. En lo que se refiere al saneamiento, 35\% de la cobertura es deficiente en las urbes latinoamericanas. Tanto es así que, en términos absolutos, la cantidad de personas sin saneamiento adecuado ha aumentado de 68 millones en 1990 a 74 millones en 2010, a pesar del incremento relativo de cobertura (onU-Hábitat, 2012, pp. 81-83).

\begin{tabular}{|l|c|c|c|c|}
\hline \multirow{2}{*}{ MEGAURBES } & \multicolumn{2}{|c|}{ 2000 } & \multicolumn{2}{c|}{ 2OIO } \\
\cline { 2 - 5 } & ZONA CENTRAL & PERIFERIA & ZONA CENTRAL & PERIFERIA \\
\hline Ciudad de México & 0,03 & 16,68 & 0,006 & 30 \\
\hline São Paulo & 0,23 & 0,42 & 0,03 & 0,08 \\
\hline Buenos Aires & 15,6 & 17,6 & 16,4 & 14,5 \\
\hline Río de Janeiro & 0,52 & 1,01 & 0,03 & 0,81 \\
\hline
\end{tabular}

TABLA 4 | Evolución de proporción de vivienda sin servicios en la periferia urbana respecto a la zona central, 2000-2010

FUENTE: ELABORACIÓN PROPIA A PARTIR DE IBGE (2000, 20IO), INDEC (200I, 20IO) E INEGI (2000, 20IO)

De manera particular, para las cuatro megaurbes se identifica una tendencia diferenciada en el tiempo, y entre la zona central y las demarcaciones de la periferia. La Ciudad de México presenta la mayor diferencia entre la zona central y los municipios conurbados, pues mientras en las 16 alcaldías que conforman la zona central han disminuido las viviendas sin servicios básicos de infraestructura, en la periferia se da una creciente proporción de viviendas carentes de ellos. Por otro lado, las megaurbes brasileńas son las que menor diferencia presentan entre el municipio central y los conurbados, con una mejora en abastecimiento en toda la zona urbana. Destaca la megaurbe Buenos Aires, pues a pesar de tener una alta proporción de viviendas desabastecidas de servicios básicos, no se da allí una gran diferencia entre la Ciudad de Buenos Aires y los 24 partidos del Gran Buenos Aires. Adicionalmente, se observa que la periferia mejora en cuanto a dotación de servicios en contraste con la zona central, donde disminuye (tabla 4).

Desde otra perspectiva, la expansión de zonas residenciales en zonas suburbanas o periurbanas sin generación de nuevas fuentes de empleo deriva en traslados largos, y de alta demanda de tiempo y dinero, hacia las zonas de trabajo. Aunado a ello, las carencias de dotación de servicios de equipamiento motivan que la población deba acudir a la zona central o semicentral para satisfacer sus necesidades educativas, de salud y recreativas, incrementando con ello los viajes radiales metropolitanos. La alta dispersión de la urbanización también dificulta la introducción de un sistema de transporte viable y rentable para el sector privado, por lo que en ocasiones se instaura uno de muy baja calidad. En muchas urbes se termina reforzando el modelo de ciudad diseñado para lograr una movilidad a partir del automóvil, lo que profundiza 
la dependencia de los hogares de este modo de transporte y la exclusión de quienes no cuentan con él (onU-Hábitat, 2012, p. 107). Retomando la desigualdad en las urbes, el nivel socioeconómico influye en los tiempos de la movilidad. La población con menos ingresos debe ocupar más tiempo en sus desplazamientos como consecuencia de los sistemas de transporte deficientes o de la incapacidad de establecer su residencia cerca de los centros de trabajo. Ello se radicaliza en muchas nuevas zonas periféricas donde no existe alternativa de transporte público.

El deterioro del hábitat influye directamente en la calidad de vida de sus habitantes, de su productividad y, consecuentemente, en la competitividad de la urbe, así como en su desarrollo. En ese sentido, el diseño adecuado de las nuevas áreas urbanas tiene un papel fundamental en el bienestar económico y social. Al mismo tiempo, esta deficiencia del hábitat y el acceso a los servicios urbanos que en ella se demandan forma parte del derecho a la ciudad y a tener un hábitat adecuado para desarrollar el potencial de los habitantes.

\section{Conclusiones: retos para la producción social del espacio en la periferia metropolitana}

Es claro que en las cuatro megaurbes latinoamericanas se observa una paulatina y constante reducción de la densidad demográfica, ocasionada por la formación de asentamientos altamente dispersos. En el caso de las ciudades brasileńas, se registra un patrón de urbanización más segmentado que en las otras incluidas en el estudio: el denominado "salto de rana". São Paulo alcanza la mayor densidad demográfica, pero con la periferia más fragmentada espacialmente. Río de Janeiro es la megaurbe más dispersa de todas, pues, además de ser la segunda más fragmentada espacialmente, es la que presenta más demanda de suelo por habitante, además de tener una proyección de expansión superior a todas las megaurbes latinoamericanas. Por su parte, Buenos Aires se extiende de manera más continua, pero con la mayor tendencia hacia la desdensificación. Asimismo, se observa un claro patrón axial a lo largo de las importantes vías de comunicación. Finalmente, la Ciudad de México mantiene un patrón mixto con una baja densidad, pero también fragmentación espacial.

La descomunal expansión urbana de las metrópolis consideradas propicia que sus habitantes no logren constituir un proceso de percepción de la totalidad del área urbana, ya no se diga de apropiación. Las historias de vida se desenvuelven solamente en los espacios donde llevan a cabo sus actividades cotidianas, generando un fenómeno de fragmentación perceptiva de la ciudad y dificultando concebir a la urbe de manera total. La expansión creciente de las megaurbes se radicaliza frente a una tendencia al uso extensivo del suelo, lo que promueve aún más la fragmentación espacial, derivada de la creciente proliferación de conjuntos habitacionales cerrados y de las grandes vías rápidas que rompen la continuidad espacial de la traza urbana. En este caso, la dispersión también fomenta la discontinuidad de la trama vial al darse grandes espacios sin urbanizar entremezclados con las nuevas zonas urbanas. La ciudad se convierte en un espacio de transición entre los diferentes espacios de vida, dejando así de ser una urbe que se vive, para ser una que se transita. Esta práctica radicaliza aún más la fragmentación perceptiva de la ciudad. 
Las empresas inmobiliarias, en su búsqueda de maximizar sus utilidades y ofrecer bienes competitivos en el mercado, buscan los predios más baratos en los alrededores de las zonas metropolitanas. Por ello, en la gran mayoría de los casos no adquieren parcelas anexas al límite urbano, sino a una mayor distancia, donde el precio es menor. Zonas sin urbanizar y de uso principalmente rural se ven sometidas a una transformación de uso de suelo, al albergar conjuntos habitacionales construidos según los criterios de rentabilidad. Este proceso fomenta la expansión de "salto de rana”, que incrementa la zona rururbana o de transición.

El patrón de expansión urbana descrito dificulta la cohesión social, pues entorpece la interacción entre avecindados y fomenta una sociedad fragmentada. Esencialmente, como hemos visto, esto se explica por los mecanismos generados por las empresas inmobiliarias en cuanto al diseńo extendido y emplazamiento aislado de los conjuntos habitacionales. Como resultado, ante la dificultad de construir el sentido de comunidad mediante las relaciones sociales, queda sin formularse un simbolismo sobre la vida social (Martínez, 2014, p. 16), mientras tampoco se desarrolla una valoración del espacio por su uso, sino solamente por su consumo.

Los procesos tradicionales de ocupación del suelo a partir tanto de la autoconstrucción de la vivienda como del desarrollo del espacio público a partir del apoyo gubernamental, permitían un proceso de apropiación, beneficiando la construcción de un hábitat en función de las necesidades de la población residente. Ahora, con la nueva lógica de generación de los espacios mediante las empresas inmobiliarias, se ofrece un espacio 'terminado' con conjuntos unifamiliares o multifamiliares que no dan cabida a transformaciones ni al interior de la vivienda ni en el entorno. Existen pocos casos de oferta de viviendas progresivas, donde la constructora solamente entrega el pie de casa y a los propietarios se les den sugerencias de ampliación de su vivienda, reconociendo con ello la heterogeneidad de los individuos en una sociedad y favoreciendo el proceso de producción social del espacio, pues la capacidad de ajustarlo proporciona a los habitantes la capacidad de construir una familiaridad cognitiva (Martínez, 2014, p. 17).

En el interior de esta nueva periferia urbana es posible identificar masivos conjuntos habitacionales sin las condiciones necesarias para crear un barrio donde la población pueda acceder a los servicios urbanos. Ello obliga a la población a salir de dichos fraccionamientos para realizar sus actividades cotidianas, impidiéndose con ello la apropiación del espacio público en el contexto inmediato a sus viviendas y la construcción de comunidad. En ese sentido, se debe fortalecer los diseños y políticas de uso de suelo mixtos, donde se favorezca la inclusión de servicios en las zonas habitacionales y se construyan espacios adecuados para ello.

A escala metropolitana, la periferia urbana ha sido el destino de la mayoría de las nuevas opciones de vivienda para una población que tiene su centro laboral en las zonas centrales o semicentrales de las zonas metropolitanas. Ello representa una movilidad residencial centro-periferia donde los nuevos residentes conservan sus espacios de vida laborales y se enfrentan a la dificultad de construir espacios de vida residenciales, por el deficiente diseńo, emplazamiento y dotación de servicios en 
dichas áreas. Por ello es que este segmento de población ${ }^{2}$ ignora el hábitat inadecuado existente en torno a su vivienda (Esquivel et al., 2005). Es decir, siguen frecuentando las zonas urbanas donde antes residían y habían construidos vínculos sociales gracias a la interacción favorecida por los espacios públicos y servicios urbanos.

Esta nueva población, cuyos espacios de vida vinculados a su lugar de residencia previos estaban relacionados con su vida cotidiana desenvuelta en otros hábitats urbanos, se enfrentan al problema de transformar sus espacios de vida situándolos en un nuevo contexto que no satisface sus necesidades básicas y se contrapone a la cotidianeidad adquirida cuando residían en su anterior vivienda. Ante la falta de servicios de equipamiento, deficiencias en la dotación de áreas verdes, la baja accesibilidad metropolitana de la nueva ubicación de su vivienda y la dificultad de adaptación del espacio privado y público a sus necesidades, no desarrollan espacios de vida en sus nuevas zonas habitacionales (Esquivel et al., 2005).

Es fundamental la regulación del uso de los nuevos espacios urbanos, la planeación territorial local y la gestión por parte de las autoridades. Las características del espacio público y las actividades cotidianas que se realizan en él son determinantes para lograr un modelo de ciudad con las condiciones de vida necesarias para lograr una urbe más equitativa e incluyente. El desarrollo local está estrechamente vinculado a la construcción de una identidad colectiva. La base del desarrollo endógeno es satisfacer las necesidades básicas de sus habitantes y promover la participación de la comunidad en dicho desarrollo mediante una visión de protección del ambiente. Por lo tanto, para lograr un desarrollo endógeno es esencial la producción social del espacio, y en especial del hábitat residencial (Carnevalli \& Trujillo, 2010, p. 21 y s.).

Los espacios bien diseñados mejoran la calidad de vida de quienes los habitan y ayudan a combatir la pobreza, al garantizar el acceso a servicios públicos. También mejoran la convivencia, al dotar a los residentes de espacios de encuentro que contribuyen a la inclusión ciudadana (Carrizosa, 2010, p. 33). No obstante, ante la nueva lógica de maximización de recursos, libertad del mercado y reducción de la intervención del Estado, se está ante un reto para la generación de una ciudad habitable frente a la tendencia a construir ciudades competitivas para el sector privado, dejando a la zaga las necesidades de la mayoría de la población.

\section{Referencias bibliográficas}

Angel, Sh., Parent, J., Civco, D., \& Blei, A. (2011). Making room for a planet of cities. Cambridge, MA: Lincoln Institute of Land Policy, Policy Focus Report. https://www.lincolninst. edu/sites/default/files/pubfiles/making-room-for-a-planet-of-cities-full_0.pdf

Arcas, J., Pagès, A., \& Casals, M. (2011). El futuro del hábitat: repensando la habitabilidad desde la sostenibilidad. El caso español. Revista INVI, 26(72), 65-93. http:// doi. org/10.4067/S0718-83582011000200003 
Augé, M. (1992). Los no lugares. Espacios del anonimato. Una antropología de la sobremodernidad. Barcelona: Gedisa.

Brites, W. (2012). Las adversidades del hábitat en conjuntos habitacionales de población relocalizada. En T. Bolívar \& J. Erazo Espinosa (coords.), Dimensiones del hábitat popular latinoamericano (pp. 121-142). Quito: Flacso Sede Ecuador; Clacso, Consejo Latinoamericano de Ciencias Sociales; Instituto de la Ciudad, Municipio del Distrito Metropolitano de Quito. http://www.biblioteca.clacso.edu.ar/ar/libros/grupos/gthi2. pdf

Carnevalli, N. \& Trujillo, A. (2010). La acción colectiva en los asentamientos informales para la transformación del hábitat. Provincia, (23), 11-31. https://www.redalyc.org/articulo. oa?id $=55516107002$

Carrizosa, M. (2010). Calidad de hábitat y responsabilidad profesional. Conformación y gestiones de la Comisión Calidad de Hábitat de la Sociedad Colombiana de Arquitectos. DEARQ: Revista de Arquitectura de la Universidad de los Andes, (6), 32-43. http:// doi.org/10.18389/dearq6.2010.05

Corzo, J. (2012). Barrios populares; hacia la búsqueda de la producción social del hábitat en Bogotá. Revista Bitácora Urbano Territorial, 20(1), 145-156. https://www.redalyc.org/ pdf/748/74824041014.pdf

Cruz, F. \& Isunza, G. (2017). Construcción del hábitat en la periferia de la Ciudad de México. Estudio de caso en Zumpango, EURE, 43 (129), 187-207. http:// doi.org/10.4067/ S0250-71612017000200009

De Mattos, C. (1998). Reestructuración, crecimiento y expansión metropolitana en las economías emergentes latinoamericanas. Economía, Sociedad y Territorio, 1(4), 723754. http://doi.org/10.22136/est001998538

De Mattos, C. (2006). Modernización capitalista y transformación metropolitana en América Latina: cinco tendencias constitutivas. América Latina: cidade, campo e turismo, (1), 41 73. http://biblioteca.clacso.edu.ar/clacso/coediciones/20100729080735/03mattos. pdf

De Mattos, C. (2008). Globalización, negocios inmobiliarios y mercantilización del desarrollo urbano. Producción inmobiliaria y reestructuración metropolitana en América Latina, 11. https://www.flacsoandes.edu.ec/agora/globalizacion-negocios-inmobiliarios-ymercantilizacion-del-desarrollo-urbano

Dear, M. \& Leclerc, G. (2013). Postborder city: Cultural spaces of Bajalta California. New York: Routledge.

Delabarre, M. \& Marry, S. (2012). Habitabilité et nature urbaines: vers un outil d'évaluation des projets urbains. Exemple de la métropole lyonnaise. Vertigo-la revue électronique en sciences de l'environnement [online], 12(2). http://doi.org/10.4000/vertigo.12683

Ducci, M. (1998). Santiago, ¿̨una mancha de aceite sin fin? ¿Qué pasa con la población cuando la ciudad crece indiscriminadamente? EURE, 24(72), 85-94. http://doi.org/10.4067/ S0250-71611998007200005

Esquivel, M. T., Maya, E., \& Cervantes, J. (2005). La promoción privada y los grandes conjuntos habitacionales: nuevas modalidades de acceso a la vivienda. Scripta Nova, 9(194). http://www.ub.edu/geocrit/sn/sn-194-21.htm 
Ewing, R. (2008). Characteristics, causes, and effects of sprawl: A literature review. En J. Marzluff, E. Shulenberger, W. Endlicher, M. Alberti, G. Bradley, C. Ryan, C. ZumBrunnen \&, U. Simon (eds.), Urban Ecology (pp. 519-535). Boston, MA: Springer. https://doi.org/10.1007/978-0-387-73412-5_34

Guillain, R., Le Gallo, J., \& Boiteux-Orain, C. (2006). Changes in spatial and sectoral pattern of employment in Ile de France, 1978-97. Urban Studies, 43(11), 2075-2098. https:// doi.org/10.1080\%2F00420980600945203

Harvey, R. \& Clark, W. (1965). The nature and economics of urban sprawl. Land Economics, 41(1), 1-9. https://doi.org/10.2307/3144884

Heidegger, M. (1994). Conferencias y artículos. Barcelona: Ediciones del Serbal.

Hernández, M. (2008). Procesos informales del espacio público en el hábitat popular. Revista Bitácora Urbano Territorial, 2(13), 109-116. https://revistas.unal.edu.co/index.php/ bitacora/article/view/18525

Hernández, V., Ocón, B., \& Guillén, J. (2009). Espacios periurbanos, transición de la ciudad al campo. Ecosostenible, (49), 5-12.

Herzog, L. (2015). Global suburbs. Urban sprawl from the Rio Grande to Rio de Janeiro. New York: Routledge.

Hidalgo, R., Borsdorf, A., Zunino, H., \& Álvarez, L. (2008). Tipologías de expansión metropolitana en Santiago de Chile: precariópolis estatal y privatópolis inmobiliaria. Scripta Nova, 12(270), 113. http://www.ub.edu/geocrit/-xcol/434.htm

Inostroza, L., Baur, R., \& Csaplovics, E. (2013). Urban sprawl and fragmentation in Latin America: a dynamic quantification and characterization of spatial patterns. Journal of Environmental Management, 115, 87-97. https://doi.org/10.1016/j. jenvman.2012.11.007

Instituto Brasileiro de Geografia e Estatística (IBGE). (2000). Censo Demográfico 2000. Rio de Janeiro: IBGE. https://biblioteca.ibge.gov.br/pt/bibliotecacatalogo?view=detalhes\&id=783

Instituto Brasileiro de Geografia e Estatística (IBGE). (2010). Censo Demográfico 2010. Rio de Janeiro: IBGE. https://censo2010.ibge.gov.br/

Instituto Nacional de Estadística y Censos (INDEC), Argentina. (2001). Censo nacional de población, hogares y vivienda, 2001. Buenos Aires: INDEC. https://www.indec.gob.ar/ micro_sitios/webcenso/index.asp

Instituto Nacional de Estadística y Censos (INDEC), Argentina. (2003). ¿Qué es el Gran Buenos Aires? Instituto Nacional de Estadística y Censos, Buenos Aires: INDEC. https://www. indec.gob.ar/dbindec/folleto_gba.pdf

Instituto Nacional de Estadística y Censos (INDEC), Argentina. (2010). Censo nacional de población, hogares y viviendas, 2010, Buenos Aires: INDEC. https://www.indec.gob.ar/ indec/web/Nivel4-Tema-2-41-135

Instituto Nacional de Geografía y Estadística (INEGI), México. (1990). Censo general de población y vivienda 1990. Aguascalientes: INEGI. https://www.inegi.org.mx/programas/ ccpv/1990/

Instituto Nacional de Geografía y Estadística (INEGI), México. (2000). Censo general de población y vivienda 2000. Aguascalientes: INEGI. https://www.inegi.org.mx/programas/ ccpv/2000/ 
Instituto Nacional de Geografía y Estadística (INEGI), México. (2010). Censo de población $y$ vivienda 2010. Aguascalientes: INEGI. https://www.inegi.org.mx/programas/ ccpv/2010/

Jiménez, W. (2013). Hábitat y vulnerabilidad, reflexiones desde lo conceptual. Revista Luna Azul, (37), 196-218. http://www.scielo.org.co/scielo.php?pid=S190924742013000200013\&script=sci_abstract\&tlng=pt

Jingnan H., Lu, X. X., \& Sellers, J. (2007). A global comparative analysis of urban form: applying spatial metrics and remote sensing. Landscape and Urban Planning, 82(4), 184-197. https://doi.org/10.1016/j.landurbplan.2007.02.010

Lang, R. (2003). Edgeless cities: exploring the elusive metropolis. Washington, DC: Brookings Institution Press.

Martínez, E. (2014). Configuración urbana, habitar y apropiación del espacio. XIII Coloquio Internacional de Geocrítica. El control del espacio y los espacios de control (pp. 1-21). Barcelona, Universitat de Barcelona, 5-10 de mayo de 2014. http://www.ub.edu/ geocrit/coloquio2014/Emilio\%20Martinez.pdf

Maya, E. \& Cervantes, J. (1999). La producción de vivienda del sector privado y su problemática en el municipio de Ixtapaluca, Estado de México. México, DF: Universidad Nacional Autónoma de México.

Maya, E., Staines, E., Roux, R., Montalvo, S., Olivares, M., Herrera, R., Tapia, R., Larenas, J., Rengifo, C., \& Cuesta, A. (2013). Marco conceptual. Marco institucional. En E. Staines (coord.), Habitabilidad y eficiencia energética en conjuntos habitacionales de interés social (pp. 17-65). Ciudad Juárez: Universidad Autónoma de Ciudad Juárez.

Nechyba, Th. \& Walsh R. (2004). Urban Sprawl. Journal of Economic Perspectives, 18(4),177200. http://dx.doi.org/10.1257/0895330042632681

Nivón, E. (2016). La Ciudad de México vista desde la periferia o la ingobernabilidad de la megalópolis. Ponto Urbe. Revista do núcleo de antropologia urbana da USP, (18), 1-16. https://journals.openedition.org/pontourbe/3063

onu-Hábitat (2012). Estado de las ciudades de América Latina y el Caribe 2012. Rumbo a una nueva transición urbana. Nairobi: Programa de las Naciones Unidas para los Asentamiento Humanos. http://www.zaragoza.es/contenidos/medioambiente/onu/ newsletter12/887_spa.pdf

Peiser, R. (2001). Decomposing urban sprawl. Town Planning Review, 72(3), 275-297. https:// doi.org/10.3828/tpr.2001.72.3.275

Perahia, R. (2009). Las transformaciones socioterritoriales en las Áreas metropolitanas. Nuevas configuraciones espaciales en la región metropolitana de Buenos Aires. 12do Encuentro de geógrafos de América Latina. Caminando en una América Latina en transformación. Montevideo: Observatorio Geográfico de América Latina. http:// observatoriogeograficoamericalatina.org.mx/egal12/Geografiasocioeconomica/ Geografiaurbana/143.pdf

Picorelli, P., Barros, G., Tomas, M., \& Molle, C. (2009). Metropolitan Regions. Working Paper ( $2^{\mathrm{a}}$ ed.). Barcelona: Metrópolis.

Pradilla, E. (2017). Teoría sobre el sprawl y ciudad compacta. De la ciudad compacta a las periferias dispersas en México. En J. Fitch, A. Escobar, \& C. Marmolejo (eds.), Ciudad y territorio: ciudad compacta vs. Ciudad dispersa. Visiones desde México y España (pp. 2747), Monterrey: Universidad Autónoma de Nuevo León. 
Prévôt, M. (2001). Fragmentación espacial y social: conceptos y realidades. Perfiles Latinoamericanos, 10(19), pp. 33-56. https://perfilesla.flacso.edu.mx/index.php/ perfilesla/article/view/315

Prévôt, M. (2002). Buenos Aires en los años 90: metropolización y desigualdades. EURE, 28(85), 31-50. http://dx.doi.org/10.4067/S0250-71612002008500003

Prévôt, M. \& Cattaneo, R. (2008). Buenos Aires: la fragmentación en los intersticios de una sociedad polarizada. EURE, 34(103), 73-92. http://doi.org/10.4067/S025071612008000300004

Robinson, J., Scott, A., \& Taylor, P. (2016). Working, housing: urbanizing. The international year of global understanding - IYGU. Cham, Switzerland: Springer. http://dx.doi. org/10.1007/978-3-319-45180-0

Rodríguez, J. (2008). Movilidad cotidiana, desigualdad social y segregación residencial en cuatro metrópolis de América Latina. EURE, 34(103), 49-71. http://doi.org/10.4067/ S0250-71612008000300003

Rodríguez, J. \& Arriagada, C. (2004). Segregación residencial en la ciudad latinoamericana. EURE, 29(89), 5-24. http://doi.org/10.4067/S0250-71612004008900001

Soja, E. (1992). Inside exopolis: scenes from Orange County. En M. Sorkin (ed.), Variation on a theme park: the new American city and the end of public space (pp. 94-122). New York: Hill \& Wang.

Thuillier, G. (2005). El impacto socio-espacial de las urbanizaciones cerradas: el caso de la Región Metropolitana de Buenos Aires. EURE, 31(93), 5-20. http:// doi.org/10.4067/ S0250-71612005009300001

Titman, Sh. (1985). Urban land prices under uncertainty. American Economic Associations, 75(3), 505-514.

United Nations (UN), Department of Economic and Social Affairs, Population Division (2014). World Urbanization Prospects: the 2014 Revision, Highlights (ST/ESA/SER.A/352). New York: United Nations. http://cdn.plataformaurbana.cl/wp-content/uploads/2015/11/ wup2014-highlights.pdf

Zamanillo, M. (2014). La producción material y la construcción social del espacio urbano. Tensiones y contradicciones. Erasmus, 16(2), 135-160. https://www.icala.org.ar/ erasmus/Archivo/2014/Erasmus\%20\%202\%20-\%202014\%20texto\%20interior.pdf

Zulaica, L. \& Celemín, J. P. (2008). Análisis territorial de las condiciones de habitabilidad en el periurbano de la ciudad de Mar del Plata (Argentina), a partir de la construcción de un índice y de la aplicación de métodos de asociación espacial. Revista de Geografía Norte Grande, (41), 129-146. http://dx.doi.org/10.4067/S0718-34022008000300007 
